\title{
Cumulative clinical experience from a decade of use: imatinib as first-line treatment of chronic myeloid leukemia
}

This article was published in the following Dove Press journal:

Journal of Blood Medicine

15 November 2012

Number of times this article has been viewed

\author{
Yusuf Baran' \\ Guray Saydam² \\ 'Department of Molecular Biology \\ and Genetics, Izmir Institute \\ of Technology, Izmir, Turkey; \\ ${ }^{2}$ Department of Hematology, \\ School of Medicine, Ege \\ University, Izmir, Turkey
}

Correspondence: Yusuf Baran Izmir Institute of Technology, Faculty of Science, Department of Molecular Biology and Genetics, Gulbahce Campus, 35430 Urla, Izmir, Turkey

Tel +902327507315

Fax +902327507509
Email yusufbaran@iyte.edu.tr

\begin{abstract}
Chronic myeloid leukemia (CML) is a malignant disease that originates in the bone marrow and is designated by the presence of the Philadelphia $(\mathrm{Ph}+)$ chromosome, a translocation between chromosomes 9 and 22. Targeted therapy against CML commenced with the development of small-molecule tyrosine kinase inhibitors (TKIs) exerting their effect against the oncogenic breakpoint cluster region (BCR)-ABL fusion protein. Imatinib emerged as the first successful example of a TKI used for the treatment of chronic-phase CML patients and resulted in significant improvements in response rate and overall survival compared with previous treatments. However, a significant portion of patients failed to respond to the therapy and developed resistance against imatinib. Second-generation TKIs nilotinib and dasatinib were to have higher efficiency in clinical trials in imatinib- resistant or intolerant CML patients compared with imatinib. Identification of novel strategies such as dose escalation, drug combination therapy, and use of novel BCR-ABL inhibitors may eventually overcome resistance against BCR-ABL TKIs. This article reviews the history of CML, including the treatment strategies used prediscovery of TKIs and the preclinical and clinical data obtained after the use of imatinib, and the second-generation TKIs developed for the treatment of CML.
\end{abstract}

Keywords: drug resistance, tyrosine kinase inhibitors, chronic myeloid leukemia, imatinib, $\mathrm{BCR} / \mathrm{ABL}$

\section{Introduction}

Chronic myeloid leukemia (CML) was first noticed in 1845 by Inokuchi, ${ }^{1}$ who realized that the disease originated in the bone marrow and used the term "myeloid leukemia." However, the pathogenesis of the disease was only understood in 1960 when Nowell and Hungerford discovered the major cause of CML, the Philadelphia ( $\mathrm{Ph}$ ) chromosome, and its relation to CML development. ${ }^{2}$ In their study, Nowell and Hungerford compared leukemia cells from chronic phase CML patients with the cells from other leukemias, and they found that the leukemic cells of chronic phase CML patients had a minute chromosome "abnormality" which was named the Philadelphia chromosome. ${ }^{3}$ In 1972, Janet Rowley demonstrated that the Ph chromosome was generated by a translocation between the long arms of chromosome 9 and chromosome $22 \mathrm{t}(9 ; 22)(\mathrm{q} 34 ; \mathrm{q} 11)$, using a specific staining procedure. ${ }^{2}$ This translocation results in the replacement of the first exon of the cellular ABL nonreceptor tyrosine kinase gene with sequences from the cellular BCR (breakpoint cluster region) gene. ${ }^{2}$ The BCR-ABL fusion oncogene was found to be encoded into a functional protein, P210 BCR-ABL, that was different from the cellular c-ABL protein in terms of its cellular location and tyrosine kinase activity. ${ }^{4,5}$ Unlike ABL which is located in the nucleus and expressed when needed, BCR-ABL fusion protein is localized in the cytoplasm of $\mathrm{Ph}+$ cells and has constitutive tyrosine 
kinase activity without any regulation. ${ }^{6}$ BCR-ABL kinase includes several different domains with important functions, such as coiled-coil motif (oligomerization domain); the tyrosine 177 (Grb-2 binding site) and rho-GEF domains on the BCR portion; and the regulatory Src homology ( $\mathrm{SH})$ regions $\mathrm{SH} 3$ and $\mathrm{SH} 2$, the $\mathrm{SH} 1$ (tyrosine kinase domain), the nuclear localization domain, DNA-binding domain, and actinbinding domain on the ABL portion. ${ }^{1,7}$ BCR-ABL activates many intracellular signaling pathways that are responsible for proliferation, genetic instability, suppression of apoptosis, and weakening of cellular adhesion. ${ }^{8,9}$ The most important pathways involved in BCR-ABL-mediated CML are Ras, STAT, PI3-K/Akt, NFкB, and Myc. ${ }^{1}$ Pendergast et $\mathrm{al}^{10}$ showed the role of the tyrosine 177 domain of BCR-ABL in BCR-ABLinduced oncogenesis. Tyr177 functions as a binding site for the SH2 domain of GRB2, which recruits SOS (son of sevenless); then, Ras is constitutively activated. ${ }^{9}$ Conversion of Tyr 177 to phenylalanine by a point mutation mostly inactivates binding of GRB2 and prevents BCR-ABL-induced Ras activation., ${ }^{70}$ Signal Transducer and Activator of Transcription (STAT) pathway, especially STAT1 and STAT5, is also constitutively activated in BCR-ABL-positive cell lines and in primary CML cells. ${ }^{11,12}$ BCR-ABL directly activates STAT1 and STAT5 independently of prior Jak phosphorylation. Activated STAT proteins trigger the expression of antiapoptotic genes such as Bcl-X1. ${ }^{13}$ The PI3-K/Akt pathway is significant for the proliferation of BCR-ABL-positive cells. ${ }^{14}$ It has been indicated that BCR-ABL activates PI3-K by forming multimeric complexes with PI3-K and adaptor protein Crkl. ${ }^{15,16}$ Activated PI3-K then converts PIP2 to PIP3 via phosphorylation. PIP3 in turn activates Akt, which regulates the function of important molecules such as the proapoptotic molecule BAD. ${ }^{17}$ Phosphorylated BAD becomes inactive because it loses its ability to bind antiapoptotic proteins such as Bcl-Xl. ${ }^{17}$ Another pathway included in CML development is the Myc pathway. Myc is activated by BCR-ABL via its SH2 domain. The pathway between the SH2 domain of BCR-ABL and Myc is thought to be related to Ras/Raf, cylin-dependent kinases, and E2F transcription factors. ${ }^{18} \mathrm{NF} \kappa \mathrm{B}$ is constantly activated in many cancer cells, and its activation is considered to be related to the development of resistance against apoptosis. At least one study has reported that CML cells with BCRABL overexpress the p65 (RelA), active subunit of NFKB. ${ }^{19}$ In addition, constant NFKB activation has been found to be necessary for generation of tumors, by the transformation of hematopoietic cells with BCR-ABL, in nude mice. ${ }^{20} \mathrm{PP} 2 \mathrm{~A}$ phosphatase, a tumor suppressor, is inactivated in the blast crisis phase of CML through the upregulation of its inhibitor,
SET, by BCR-ABL. Inactivated PP2A causes the hyperphosphorylation and inactivation of BAD. As a result, CML cells escape apoptosis. ${ }^{21}$

\section{Clinical presentation and stages of CML}

The disease, whose clinical presentations are insidious, is often discovered incidentally in the chronic phase, when an elevated white blood cell count is revealed by a routine blood count or when an enlarged spleen is found on a general physical examination. The most common findings are as follows for patients who present with symptoms: fatigue/ lethargy, bleeding episodes due to platelet dysfunction (for eg, purpura; menorrhagia; prolonged bleeding after dental extraction; and other mucosal bleeding, such as epistaxis), weight loss, anorexia, excessive sweating, abdominal fullness or pain because of splenic pathology, and malaise. ${ }^{22}$ Additionally, patients often present with physical findings of splenomegaly, which is the most common physical finding in patients with CML. However, hepatomegaly, which is usually part of the extramedullary hematopoiesis occurring in the spleen, is unusual. Involvement of extramedullary tissues, including the lymph nodes, soft tissues, and skin, is generally limited to patients presenting after progression to acute leukemia. $^{23}$

\section{Stages of CML}

CML has a multiphasic clinical course that consists of a chronic, accelerated, and blast phase. Accelerated and blast phases occur at a median of 3-5 years after disease onset.

\section{Chronic phase}

The chronic phase is observed at the time of diagnosis in approximately $85 \%$ to $90 \%$ of patients. The chronic phase can be defined as a genetically unstable state. Accumulation of additional molecular and chromosomal abnormalities results in impairment of hematopoietic differentiation and disease transformation, and a high proliferative rate during this stage allows for this. ${ }^{24}$

\section{Accelerated phase}

Accelerated phase of CML is diagnosed if one or more of the following criteria is observed: (1) blasts comprise $10 \%$ to $19 \%$ of the peripheral blood white cells or bone marrow cells; (2) peripheral blood basophils are at least 20\%; (3) persistent thrombocytopenia $\left(<100 \times 10^{9} / \mathrm{L}\right)$ unrelated to therapy, or persistent thrombocytosis $\left(>1000 \times 10^{9} / \mathrm{L}\right)$ unresponsive to therapy; (4) increasing spleen size and increasing white blood cell (WBC) count unresponsive 
to therapy; (5) cytogenetic evidence of clonal evolution; (6) megakaryocytic proliferation in sizable sheets and clusters, associated with marked reticulin or collagen fibrosis; and/or severe granulocytic dysplasia. ${ }^{24}$

\section{Blast phase}

The accumulation of chromosomal abnormalities and uncontrolled differentiation eventually causes acute leukemia, or in other words, blast-phase CML. This phase is usually identified by similar symptoms as in the accelerated phase. Blast phase of CML is diagnosed if one or more of following criteria is observed: (1) blasts comprise $20 \%$ or more of the peripheral blood white cells or bone marrow cells; (2) extramedullary blast proliferation is observed; and (3) large foci or clusters of blasts are found in bone marrow biopsy. $^{24}$

\section{Preimatinib treatment strategies of $\mathrm{CML}$}

Before the imatinib era, interferon (IFN)- $\alpha$-based treatment and stem cell transplantation were the treatments of choice in newly diagnosed patients.

\section{IFN- $\alpha$}

IFN- $\alpha$, which was discovered in the 1980s, was the first pharmacological treatment that substantially affected the disease's natural course. Unlike traditional cytoreductive chemotherapies, IFN- $\alpha$ had the ability to induce complete cytogenetic remission in varying frequencies (as high as 26\%) in chronic-phase patients and allowed prolonged survival. ${ }^{25}$ Nevertheless, side effects, such as fatigue, myalgias, arthralgias, headaches, weight loss, depression, diarrhea, neurological symptoms, memory changes, hair thinning, autoimmune diseases, and cardiomyopathy, limited the clinical utility of interferon- $\alpha .^{26}$

Clarification of the anti-CML effect of IFN-alpha opened new scientific windows including determination of optimized dosing, evaluation of PEGylated interferon- $\alpha$, and combination with other substances like Cytarabine. ${ }^{27}$

\section{Allogeneic stem cell transplantation}

Allogeneic stem cell transplantation (SCT) is the treatment modality most often used to treat CML patients. The advantage of SCT is encumbered by side effects that include immunodeficiency, infections, organ toxicity (from the conditioning regimen), and acute and chronic graft-versushost disease, and which cause significant cure-associated mortality. The efficiency of allogeneic SCT for CML cure is mainly associated with alloimmune effects, as indicated by the excellent results obtained with donor lymphocyte infusions in the event of posttransplant relapse. ${ }^{28}$

\section{Preclinical development of imatinib and in vitro studies}

Understanding the molecular mechanisms underlying CML and the significant role of BCR-ABL tyrosine kinase activity in cancer progression has led to the development of targeted therapies. ${ }^{29}$ In the early 1990 s, scientists under the supervision of $\mathrm{N}$ Lydon and A Matter at Ciba Geigy (now Novartis), studied the development of specific tyrosine kinase inhibitors, which resulted in the discovery of a firstgeneration tyrosine kinase inhibitor (TKI) called imatinib. This compound was a 2-phenylaminopyrimidine derivate and was initially generated as a relatively weak inhibitor of protein kinase $\mathrm{C}$-alpha $(\mathrm{PKC} \alpha)$ and the platelet derived growth factor receptor (PDGFR). ${ }^{30,31}$ The first derivatives of the 2-phenylaminopyrimidine series had low water solubility. Therefore, they had poor oral bioavailability. The addition of N-methylpiperazine, a highly polar side chain, significantly increased solubility and oral bioavailability, and the most potent molecules inhibited both $\mathrm{v}$-Abl and the PDGFR kinases. Imatinib mesylate (formerly STI571, now Gleevec ${ }^{\circledR}$ or Glivec $^{\circledR}$; Novartis, Basel, Switzerland) emerged as the first successful example of a BCR-ABL tyrosine kinase inhibitor used for the treatment of CML, since it selectively inhibited the growth of BCR-ABL-positive cells. ${ }^{32}$

Imatinib binds to the adenosine triphosphate (ATP) binding pocket of the inactive conformation of the ABL protein tyrosine kinase and prevents its switch to the active form. ${ }^{33}$ Submicromolar concentrations of imatinib were shown to inhibit autophosphorylation of various protein tyrosine kinases, such as $\mathrm{V}-\mathrm{Abl}$, c-Kit receptor (the receptor for stem cell factor), PDGFR-A and B, and the Abelsonrelated gene (ARG).$^{34}$ It also blocked PDGF-induced inositol phosphate formation, mitogen-activated protein (MAP) kinase activation, and c-fos mRNA expression in intact cells. ${ }^{35,36}$ In contrast, the compound was inactive against signal transduction mediated by insulin, insulin-like growth factor 1 (IGF-1), epidermal growth factor (EGF), and phorbol ester. ${ }^{36}$ Additionally, imatinib did not inhibit the receptor for colony-stimulating factor 1 (CSF-1) or the nonreceptor tyrosine kinases family of Src.

Several studies have reported the antiproliferative activity of imatinib against a variety of cell lines expressing activated ABL proteins. ${ }^{34}$ The growth-inhibitory effect of imatinib was confirmed in various CML and $\mathrm{Ph}+\mathrm{ALL}$ cell lines, ${ }^{37-40}$ whereas no effect of imatinib was observed on 
$\mathrm{Ph}$ - cell lines. ${ }^{37,39}$ In an in vitro study, imatinib was shown to decrease CML colony growth by $92 \%-98 \%$ without significantly inhibiting normal colony growth. ${ }^{41}$ Incubation of cells with imatinib resulted in apoptotic cell death. ${ }^{34,39}$

Furthermore, increasing evidence has suggested that imatinib mesylate affects the development and function of antigen-presenting cells, such as dendritic cells and $\mathrm{T}$ cells. ${ }^{42}$ However, the exact nature of the effects of imatinib (activation or suppression) on immune cells has to be further discovered.

The activity of imatinib has also been tested in vivo. BCRABL- and v-Src-transformed 32D cells were introduced into syngeneic mice separately, and increasing doses of imatinib were given intraperitoneally. Dose-dependent inhibition was observed in BCR-ABL-transformed tumors while intraperitoneal treatment of imatinib exerted no growth-inhibitory effect on tumors derived from v-Src-transformed 32D cells, consistent with the lack of inhibition of Src kinase activity by imatinib. ${ }^{34}$ In another study, imatinib was demonstrated to have antitumor effects in $\mathrm{p} 210^{\mathrm{BCR}-\mathrm{ABL}}$-expressing cells, whereas no growth inhibition was observed in groups of mice injected with a BCR-ABL-myeloid cell line, U937. In addition, imatinib was also tested in lethally irradiated syngeneic mice that received marrow infected with a BCR-ABL retrovirus (also called a transduction-transplantation model of CML). ${ }^{43,44}$ Results showed that treatment with imatinib led to prolonged survival; ${ }^{45}$ however, responses were variable, suggesting formation of a trend toward "clonal depletion" where imatinib was able to successfully target some leukemic clones, but not all.

\section{Clinical introduction of imatinib and the results of clinical use of imatinib}

The clinical introduction of imatinib started with a phase I clinical trial that recruited patients with chronic phase CML who had failed therapy with IFN- $\alpha .{ }^{46}$ At doses greater than $300 \mathrm{mg}$, very impressive clinical outcomes were detected, with 53 of 54 patients obtaining a complete hematological response (CHR) (disappearance of clinical and hematological sign and symptoms of the disease) and 31\% achieving major cytogenetic response (MCyR) (0\%-35\% $\mathrm{Ph}+$ metaphases), including a complete cytogenetic response (CCyR) rate of $13 \%$. Importantly, the responses were durable, with only two of 53 patients relapsing during a median follow-up of 265 days. For further studies, a daily dose of $400 \mathrm{mg}$ was selected based on pharmacokinetic data confirming that this provided a mean plasma concentration greater than needed to suppress BCR-ABL1 activity. To clarify the activity of imatinib against blast phase CML and relapsed/refractory $\mathrm{Ph}+$ acute lymphoblastic leukemia (Ph+-ALL), the phase I study was expanded to include these patients. ${ }^{47}$ Single agent therapy with imatinib resulted in CHR in $11 \%$ of patients with myeloid blast phase and $20 \%$ of patients with lymphoid blastic disease. Some of the remaining patients showed reduction in the blast count, but unfortunately, responses were short lived, and most of the patients showed rapid progression. In contrast to patients treated in the chronic phase, almost $50 \%$ of responders with myeloid disease and all but one responder with lymphoid disease relapsed between 42 days and 193 days of imatinib therapy. ${ }^{47}$

After the phase I trials, three international phase II trials were started in 1999. These studies enrolled patients with CML who had failed IFN- $\alpha$; patients with CML in myeloid blast crisis; patients with relapsed Ph-positive ALL; and patients with CML in accelerated phase. The results obtained from patients with CML in myeloid blast crisis largely confirmed the results seen in the phase I study. ${ }^{48}$ However, it is difficult to conclude how the results of imatinib compare with conventional chemotherapeutic agents, since no controlled trials were available for this population. But CHR was observed in $15 \%$ of patients; sustained response over 4 weeks was seen in $31 \%$ of patients; MCyR was seen in $16 \%$ of patients; and the median survival was 6.8 months. Additionally, the one year survival rate, $30 \%$, was better than in any previously reported study. ${ }^{49-51}$ Also, imatinib provided some benefits in terms of oral administration and use in outpatient settings, which resulted in better quality of life.

Contrary to the response in patients with myeloid blast crisis, there have been almost no durable responses in blast crisis with a lymphoid phenotype and in $\mathrm{Ph}$-positive acute lymphoblastic leukemia with the exception of patients treated due to relapse after an allograft. ${ }^{52,53}$ Although there were no patients with accelerated phase in the phase I protocols, not surprisingly, the results for this group are intermediate to those observed in myeloid blast crisis and chronic phase. ${ }^{54}$ For patients in the accelerated phase, CHR, MCyR, and CCyR were reported as $53 \%, 24 \%$, and $17 \%$, respectively, with $74 \%$ estimated survival at 12 months. This 1 -year, estimated survival rate is twice as high as the previously published study with the best results. ${ }^{51}$ Additionally, $17 \%$ of patients treated with imatinib obtained CCyR, which was very rare in treatment of the accelerated phase with other therapies. But this lasted for a very short time period which indicated the need for further strategies for this group of patients. The accelerated-phase study was started with an imatinib dose of $400 \mathrm{mg}$ per day that was increased to $600 \mathrm{mg}$ per day 
when safety data were obtained. The higher daily dose of $600 \mathrm{mg}$ imatinib resulted in a significantly longer time to progression and better overall survival (OS) compared with the $400 \mathrm{mg}$ cohort.

The largest of the phase II trials enrolled patients in chronic phase who had been previously treated with IFN$\alpha$-based therapies and failed. Patients were stratified as hematologically, cytogenetically refractory or resistant; and intolerant to IFN- $\alpha$. The rate of complete hematologic response in all patients was $95 \%$, with $89 \%$ progression-free survival at 18 months. Moreover, the rate of CCyR was $41 \%$ and $60 \% \mathrm{MCyrR}$. One of the most interesting findings in this study was that the best results were obtained in patients who had previously achieved a cytogenetic response to IFN- $\alpha$. This effect of IFN was also noticed in the 2000s, with the development of newer TKIs and many clinical studies combining TKI and IFN- $\alpha$ to cure CML.

Randomized phase III trials were conducted to compare IFN- $\alpha$ plus cytarabine versus IFN- $\alpha$ alone ${ }^{55}$ and imatinib with IFN- $\alpha$ plus cytarabine ${ }^{56}$ in CML patients. At the start of this study, the combination of IFN and cytarabine was accepted as the best nontransplant treatment strategy for $\mathrm{CML}^{55}$ Results obtained from the phase III study showed that imatinib was notably superior in terms of the rates of CHR, MCyR, and CCyR. Moreover and most importantly, there was a significant difference between two arms favoring imatinib in terms of progression to accelerated and blastic phase at 18 months. ${ }^{56}$ Based on these results, the FDA approved imatinib as a first-line treatment for newly diagnosed CML in December 2002. This study was the most important study in the historical course of CML and is known as the IRIS study (International Randomized Study of IFN and STI571). The IRIS trial randomized 1106 patients with newly diagnosed CML to imatinib (400 mg daily) and IFN- $\alpha$ plus low-dose cytarabine. ${ }^{56}$ Early results showed the superiority of the imatinib treatment arm compared to other arm, with MCyR rate of $87 \%$ versus $35 \%(P<0.001)$, and CCyR of $76 \%$ versus $15 \%(P<0.01)$, respectively. Transformation-free survival (TFS) evaluated at 18 months showed the benefit of imatinib, 97\% versus $91.5 \%(P<0.001) .{ }^{56}$ Imatinib was also found to be much better tolerated compared with IFN- $\alpha+$ cytarabine. Only $3 \%$ of patients treated with imatinib in this study discontinued therapy due to adverse effects or crossed over to the other treatment arm because of intolerance while $30 \%$ of patients treated with IFN- $\alpha+$ cytarabine crossed over to the imatinib arm due to intolerance. Although adverse events, including superficial edema, nausea, muscle cramps, and rashes, were more commonly seen in the imatinib arm, most of these were grade 1 or 2 . The most reported grade 3 or
4 adverse events were cytopenias, anemia, neutropenia, and thrombocytopenia and were more frequent in the IFN- $\alpha+$ cytarabine treatment arm. ${ }^{57}$

Long-term follow-up results of the IRIS trial have confirmed the benefits of imatinib and the durable response of the drug if continued. After 8 years, 304 patients from the original cohort (55\%) remained under treatment with imatinib. ${ }^{58}$ The CCyR rate at 8 years was $83 \%$, with $18 \%$ having lost CCyR and $3 \%$ having progressed to accelerated/blast phase. Eventfree survival (EFS) was $81 \%$ and TFS was $92 \%$. The patients who obtained major molecular response (MMR) (defined as a 3-log reduction of BCR-ABL1 transcripts from a standardized baseline value, as assessed by reverse transcriptase polymerase chain reaction [RT-PCR]) at 12 months remained in TFS, with a percentage of $100 \%$ at 8 years. The rate of progression to accelerated/blast phase decreased over time by years, being $1.5 \%$ for the first year, $0 \%$ for 6 and 7 years, and $0.4 \%$ for eight years. At 8 years, OS was $85 \%$ (93\% if only CML-related deaths are considered). Since the designation of the study allowed crossover between treatment arms, there was no difference in survival between arms. However, a few studies and reports comparing cohorts of patients treated with imatinib with historical CML controls revealed that imatinib clearly improved survival in patients with CML treated with formerly standard therapies such as IFN- $\alpha+$ Ara-C. ${ }^{59-61}$

Although the long-term results and safety data of imatinib are convincing, very long-term survival benefits (OS and EFS) have only been recently reported. Kantarjian et al ${ }^{62,63}$ have published single-institution results of 368 patients with Ph-positive CML in the chronic phase who received imatinib therapy after failure on IFN- $\alpha$, with median follow up of 114 months (range 1-132 months). Researchers noted favorable results, with a 10 -year survival rate of $68 \%$ and EFS rate of $51 \% .^{62}$ These results clarify the favorable outcome of patients after IFN failure, a group whose median survival was 3 years when imatinib therapy was not available. ${ }^{63}$ In the study by Kantarjian et al, the estimated 10-year survival rate of patients was $68 \%$, compared with $20 \%$ to $30 \%$ in the historic group of patients who had failure on IFN therapy and no access to imatinib. ${ }^{63}$

\section{Recommendations for use of imatinib in the treatment of patients with CML}

The European LeukemiaNet organization has published guidelines on treatment strategies and monitoring of patients with CML and treated with TKIs. ${ }^{64}$ Based on these guidelines and recommendations, all patients with the diagnosis of CML 
should be treated with TKIs, and until the FDA approval of nilotinib and dasatinib for first-line use, the only option for this treatment is imatinib. These guidelines also clearly identify patients with an inadequate response to therapy and who require a change in treatment. ${ }^{65,66}$ Table 1 summarizes the latest version of the European LeukemiaNet guidelines.

The optimal response, which is ideal for all patients, is defined as: a CHR at 3 months, a partial cytogenetic response (PCyR) at 6 months, a CCyR at 12 months, and an MMR at 18 months. Based on these definitions, failure in treatment should be considered when a patient does not achieve a CHR at 3 months, any CyR at 6 months, a CCyR at 12 months and an MMR at 18 months. The loss of CHR or CCyR at any time or clonal evaluation under the treatment is also accepted as treatment failure. Therapy for these patients should be changed. Suboptimal response is still debated and can be understood as a response that does not meet the above mentioned criteria for optimal response or the criteria for failure. Suboptimal response represents some of the patients that respond slowly or inadequately. Clinical studies have confirmed that suboptimal responders at early time points, for example 6 months, have similar outcomes in terms of OS, PFS, and EFS as those with failure to therapy. ${ }^{67-69}$

\section{Evaluation and monitoring the response to imatinib therapy}

Response to imatinib therapy should be monitored by evaluation of hematologic, cytogenetic, and molecular status of patients at certain time points. Hematological response can

Table I Response criteria for patients treated with imatinib

\begin{tabular}{|c|c|}
\hline Response & Definitions \\
\hline \multicolumn{2}{|c|}{ Hematologic response (HR) } \\
\hline \multirow[t]{5}{*}{ Complete (CHR) } & $\mathrm{WBC}<10 \times 10^{9} / \mathrm{L}$ \\
\hline & Basophils $<5 \%$ \\
\hline & No myelocytes, promyelocytes, \\
\hline & myeloblasts in differential \\
\hline & Platelet count $<450 \times 10^{9} / \mathrm{L}$ \\
\hline \multicolumn{2}{|c|}{ Cytogenetic response (CyR) } \\
\hline Complete (CCyR) & No Ph+ metaphases \\
\hline Partial (PCyR) & $1 \%-35 \% \mathrm{Ph}+$ metaphases \\
\hline Minor (mCyR) & $36 \%-65 \% \mathrm{Ph}+$ metaphases \\
\hline Major (MCyR) & $0 \%-35 \% \mathrm{Ph}+$ metaphases \\
\hline \multicolumn{2}{|c|}{ Molecular response (MR) } \\
\hline Complete (CMR) & Undetectable BCR-ABLI mRNA \\
\hline \multirow[t]{4}{*}{ Major (MMR) } & transcripts by RT-PCR in two consecutive \\
\hline & blood samples of adequate quality \\
\hline & Ratio of $B C R-A B L / A B L<0.1$ on the \\
\hline & international scale \\
\hline
\end{tabular}

Abbreviations: WBC, white blood cells; BCR, breakpoint cluster region; ABLI, Abelson murine leukemia viral oncogene homolog I; RT, reverse transcription; $\mathrm{PCR}$, polymerase chain reaction. be assessed by complete blood count from peripheral blood every 2 weeks until a CHR is obtained and confirmed, and every 3 months thereafter.

Since it has been clearly documented that obtaining a cytogenetic response to therapy in patients with CML is associated with improved survival and decreased risk of transformation to accelerated/blast phase, cytogenetic response has been accepted as the most important criterion for evaluation of treatment in patients with CML. ${ }^{70} \mathrm{CCyR}$ is the gold standard, and bone marrow must be reassessed every 6 months until CCyR is obtained and confirmed, and at least every 12 months thereafter, if regular molecular monitoring cannot be assured. Imatinib studies have also clearly demonstrated that not only the depth of response, but also the timing of this response is very important. For example, patients who achieved CCyR at 12 months of imatinib therapy had a 5-year EFS of 97\%, while this was $93 \%$ for patients with a PCyR and $81 \%$ for those who had failed to obtain a MCyR altogether $(P<0.001)$. $^{71}$ However, since cytogenetic analysis can confirm decrease of only a small amount of leukemic clone, more sensitive techniques, to evaluate the amount of remaining leukemic clone in bone marrow, are needed. Monitoring the level of BCR-ABL1 transcript level by quantitative RT-PCR has revealed that this technique is a good method to further quantify residual disease in cases with CML, even in CCyR. Many ongoing studies are being conducted to evaluate the clinical importance of the molecular response. Based on data obtained to date from the IRIS trial, patients achieved CCyR and MMR at 18 months of treatment and had a $100 \%$ ratio of 5-year OS. ${ }^{72}$ The European Leukemia Net has defined the criteria for optimal response, suboptimal response, and failure to therapy with TKI and published a guideline for monitoring patients with CML (Tables 2 and 3). ${ }^{63}$

However, there is still debate on the importance of obtaining MMR after having CCyR. Since the longest data are from the IRIS trial, Hughes et $\mathrm{al}^{72}$ investigated the importance of MMR in this trial. At 18 months, patients who had not achieved an MMR had a significantly inferior 7 -year EFS (95\% vs 75\%, $P<0.001$ ) and TFS (99\% vs 90\%, $P<0.001)$. While there was no difference in OS $(95 \%$ vs $90 \%$ ) when only the patients who achieved a CCyR were analyzed, obtaining an MMR at 18 months was associated with a lower rate of loss of CCyR (3\% vs $26 \%, P<0.001)$ and a better 7-year EFS (95\% vs $86 \%$ ) but no improvement in TFS (99\% vs $96 \%$ ) or OS (95\% vs 96\%). Thus, the results clearly indicate that although obtaining MMR is beneficial for patients with CML, CCyR is still the only surrogate marker associated with improvement in OS. Also, it should not be 
Table 2 The definitions of response criteria for patients with CML ${ }^{63}$

\begin{tabular}{lll}
\hline Hematologic & Cytogenetic & Molecular \\
\hline Complete: Normal CBC, nonpalpable spleen, & Complete: $0 \% \mathrm{Ph}+$ metaphases & Complete: Undetectable BCR-ABLI transcripts \\
and disappearance of all disease signs and symptoms & Partial: $1 \%-35 \% \mathrm{Ph}+$ metaphases & on two consecutive qRT-PCR or nested PCR \\
& Major: $0 \%-35 \% \mathrm{Ph}+$ metaphases & assay (sensitivity at least $10^{-4}$ ) \\
& Minor: $36 \%-65 \% \mathrm{Ph}+$ metaphases & \\
& Minimal: $66 \%-95 \% \mathrm{Ph}+$ metaphases & \\
& No response: $\geq 96 \% \mathrm{Ph}+$ metaphases & \\
\hline
\end{tabular}

Abbreviations: CML, chronic myeloid leukemia; CBC, complete blood count; BCR, breakpoint cluster region; ABLI, Abelson murine leukemia viral oncogene homolog I; $R T$, reverse transcription; PCR, polymerase chain reaction.

forgotten that failure to obtain MMR or complete molecular response have not been defined as the failure of therapy, and a couple of clinical studies have confirmed this. ${ }^{66,73}$ Based on these data, it is recommended that physicians should not decide to change the treatment of TKIs based on a 1-log increase in BCR-ABL1 transcript level, especially in patients in CCyR. Also, the increase in molecular script should be repeated to confirm results, and it is reasonable to change treatment based on loss of molecular response.

Recently, Marin et $\mathrm{al}{ }^{75}$ published an article showing the importance of early log reduction of BCR-ABL1 transcripts at 3 months of therapy. They proved that patients with a $10 \%$ $\log$ reduction in BCR-ABL1 levels at 3 months have better PFS, cumulative incidence of CCyR, and complete molecular response than those with higher transcript levels. One could assume that the evaluation of response in patients treated with TKI would be recommended earlier than in current strategies.

\section{Definition of resistance to imatinib}

Although treatment of newly diagnosed CML patients with imatinib has been defined as the standard of care for CML patients, these patients exhibit a heterogeneous sensitivity to imatinib. Primary refractoriness to imatinib is an initial insensitivity to imatinib and is represented by a small fraction of patients with primary resistance. Primary resistance in these patients is defined as the failure to obtain CHR by 3 months,

Table 3 Recommendations for monitoring patients with $\mathrm{CML}^{63}$

$\mathrm{CBC}$

Cytogenetic

Molecular

Mutation analysis

Every 2 wks until CHR; then every 3 mo or as needed During the diagnosis, $3 \mathrm{mo}, 6 \mathrm{mo}$, and every $6 \mathrm{mo}$ until CCyR; then every 12 mo, if molecular test is not available

At failure or unexpected myelosuppression

Every 3 mo until MMR; then every 6 mo

In patients with failure, suboptimal response, and before changing therapy with second generation TKIs

Abbreviations: CML, chronic myeloid leukemia; CBC, complete blood count; $\mathrm{CHR}$, complete hematologic response; CCyR, complete cytogenetic response; MMR, major molecular response; mo, months; TKIs, tyrosine kinase inhibitors.
CCyR by 12 months, and MMR by 18 months. ${ }^{75}$ The validity of this definition was demonstrated by two independent series and studies. ${ }^{68,69}$ In the IRIS study, primary resistance was found in $24 \%$ of patients at 18 -month follow up. ${ }^{56}$ In addition to primary resistance, some patients may develop secondary resistance, which is acquired and seen under imatinib treatment after an initial typical response. Secondary resistance was reported in $7 \%$ of patients, and relapsed disease developed in $17 \%$ at 5 -year follow up. ${ }^{71}$

Imatinib resistance can develop with multiple mechanisms, which can be classified as BCR-ABL1-dependent or independent. ${ }^{75}$ The most studied and characterized mechanism is the accumulation of point mutations within the kinase domain of the BCR-ABL1 gene, resulting in conformational changes and disruption of the BCR-ABL1/ imatinib interface. ${ }^{76}$ Direct sequencing techniques have revealed the presence of almost 100 different mutations in samples obtained from $50 \%$ of imatinib-resistant patients in the chronic phase. ${ }^{76,77}$ These mutations alter the binding of imatinib to its specific site or disrupt and change the structure of the binding site. Some of the most frequent mutated residues involve Y253, E255, and T315I. ${ }^{78}$ Currently, the T315I mutation, which is a gatekeeper mutation, has been proven to be the most resistant to almost all available TKIs. ${ }^{79}$ In contrast, P-loop mutations may confer relatively high resistance to imatinib that can be overcome, at least in some cases, with the use of second-generation TKIs. The T315I mutation results in resistance to all commercially available TKIs because it prevents the formation of an important hydrogen bond between TKIs and the T315 amino acid residue of the BCR-ABL1 molecule. This blocks binding of TKIs to the BCR-ABL1 protein. The T315I mutation is a common mechanism of resistance in CML patients evolving to accelerated phase or blast phase under treatment with TKI. ${ }^{77}$ Screening for mutations is currently recommended in the following situations: (a) for patients with accelerated/blast phase at diagnosis, and (b) for patients with failure or suboptimal response under therapy with imatinib or another TKI. ${ }^{80}$ 
Other mechanisms of resistance are classified as non-BCRABL1-dependent and are diverse and not well understood. Activation of signaling pathways other than BCR-ABL1 is one of the potential mechanisms for leukemic cells to escape inhibition by imatinib. For example, the activation of the Srcfamily kinase enzymes potentiates cell proliferation without activation of BCR-ABL1 ${ }^{81}$ Impairment of transportation of imatinib from the extracellular area across the cell membrane to cytoplasm is another imatinib-resistance mechanism. The human organic cationic transporter-1 (OCT-1) is the main transporter protein responsible for imatinib influx, and polymorphisms can potentially have affect expression of OCT-1. ${ }^{82}$ Patients with CML and a low level of OCT-1 have inferior levels of MMR, CMR, EFS, and OS at 5 years. ${ }^{82}$ Increasing the dose of imatinib can overcome the negative effect of a low level of OCT-1, but this strategy needs to be confirmed by further studies. Neither dasatinib nor nilotinib, secondgeneration TKIs, use OCT-1 for transport across the cell membrane, and this could be an advantage for these newer compounds. ${ }^{82}$ Additionally, both nilotinib and dasatinib have been approved for treatment of newly-diagnosed patients with CML, based on recent trials. ${ }^{83,84}$

\section{Strategies for overcoming imatinib resistance}

Cases of acquired resistance to imatinib therapy have constituted the need to develop new effective agents for inhibiting tyrosine kinase activity. A second generationTKI, nilotinib (Tasigna $^{\circledR}$; AMN107) was approved by the FDA in 2007 for overcoming imatinib resistance or intolerance in $\mathrm{Ph}+\mathrm{CML}$ patients. ${ }^{85}$ Nilotinib, which is more effective and potent than imatinib, acts on most BCR-ABL point mutations that cause imatinib resistance. ${ }^{86,87}$ Because of similarity in the structures of nilotinib and imatinib, nilotinib requires that the ABL protein be in the inactive conformation in order to bind appropriately. ${ }^{88}$ In vitro studies have indicated that nilotinib treatment of CML cells triggers apoptosis and also suppresses autophosphorylation of BCR-ABL on tyrosine 177 (Tyr177), which is an important binding site for growth factor receptor-bound protein 2 (Grb2) adapter protein. In addition, Tyr177 is an important factor in the pathogenesis of BCR-ABL, because it controls several signaling pathways from PI3K to Ras/Erk. ${ }^{89}$ It has been reported that nilotinib treatment of K562 cells inhibits the autophosphorylation activity of BCR-ABL on Tyr $177 . .^{90}$ The greater potential of nilotinib may be because it has a higher affinity to the $\mathrm{ABL}$ kinase pocket than does imatinib. This is because the connection between nilotinib and the binding surface on ABL raises and fits appropriately. ${ }^{87}$ Furthermore, two in vivo studies $^{87,88}$ showed that nilotinib considerably suppressed leukemia cell aggregation in the spleen, marrow, liver, and also lymph nodes of mice with BCR-ABL-transformed bone marrow cells. Nilotinib was also reported to increase the survival periods of mice synthesizing imatinib-resistant mutant proteins and BCR-ABL. Importantly, these in vivo studies also reported that nilotinib treatment of mice did not cause any hematopoietic toxicity. Moreover, phase I and phase II studies revealed that nilotinib was more effective than imatinib, had fewer side effects in CML patients, and increased patient survival. ${ }^{91-93}$

Dasatinib (Sprycel ${ }^{\circledR}$; BMS-354825), another TKI, is basically an inhibitor of the Src-family kinases. Dasatinib has the ability to inhibit ABL, BCR-ABL, PDGF receptor, and also Src, Fyn, and Lyk (tyrosine-protein kinases). ${ }^{94,95}$ Dasatinib binds to the ATP-binding site of ABL but lies on the opposite side from imatinib. While imatinib and nilotinib can bind only to the inactive form of $\mathrm{ABL}$, dasatinib can bind both active and inactive forms of $\mathrm{ABL}$. In addition, dasatinib binds to $\mathrm{ABL}$ through fewer interactions than imatinib and nilotinib, and its affinity for binding to ABL is higher than that of imatinib. ${ }^{88,96}$ Dasatinib was reported to have antiproliferative effects on all CML cells bearing imatinib-resistant BCRABL mutants except for those bearing the T315I mutation. ${ }^{97}$ Phase I and phase II studies revealed that the adverse effects of dasatinib, such as headache, rash, diarrhea, and edema, were fewer than those of imatinib. ${ }^{98,99}$

ON012380, another ABL inhibitor, blocks the substrate binding site of ABL rather than the ATP binding site, as imatinib does. Studies have shown that, unlike imatinib, nilotinib, and dasatinib, ON012380 can suppress both wild type $\mathrm{ABL}$ and also imatinib-resistant ABL kinase mutations, including the T315I mutation. ${ }^{88,100}$ It was reported that although ON012380 and imatinib can bind ABL regions distinct from each other, they act on the inhibition of BCRABL kinase activity in a synergistic manner; ON012380 was reported to be more effective than imatinib in the inhibition of kinase activity of wild type BCR-ABL, whereas, their effects were found to be similar in both in vitro and in vivo imatinib-resistant mutants. ${ }^{100}$ Moreover, treatment of mice with this compound revealed that it did not show any toxic effect on the organism. ${ }^{88}$

NS-187/INNO406 acts as an inhibitor for both ABL and Lyn kinase and is more effective than imatinib in vitro. Except for the T315I mutation, it is also effective on BCR-ABL kinase domain mutants. ${ }^{101}$ In vivo studies showed that NS-187/INNO406 increased survival 
periods of mice having leukemia generated by BCR-ABL expression. ${ }^{8}$

Aurora kinases, which are upregulated in many types of cancer cells, are crucial in the regulation of the cell cycle in mitotic phase. MK0457 (VX680), which is an aurora kinase inhibitor, is also an effective agent for inhibiting the other kinase-resistant and T315I mutants of BCR-ABL kinase. Studies indicated that MK0457 suppressed cellular proliferation in CML patients with T315I mutations. ${ }^{102,103}$

Farnesyl transferase inhibitors, which inhibit downstream of RAS signaling, are another approach for overcoming imatinib-resistance. Tipifarnib, an inhibitor of farnesyl transferase, was reported to be effective in imatinib-resistant CML patients when used in combination with imatinib. ${ }^{104,105}$ In addition, another farnesyl transferase inhibitor, lonafarnib, is also effective in imatinib-resistant CML patients. ${ }^{106}$

Rapamycin, an inhibitor of mammalian target of rapamycin (mTOR), is also effective on the proliferative activities of CML cells, in a synergistic manner with imatinib. Use of rapamycin or other $\mathrm{mTOR}$ inhibitors, either in combination with imatinib or alone, affects cell proliferation in imatinibresistant CML cells. ${ }^{107}$

Heat shock protein-90 (HSP90) inhibition, which results in AKT reduction and apoptosis is another strategy for overcoming imatinib-resistance. ${ }^{103}$ Celastrol, an inhibitor of HSP90, was reported to suppress BCR-ABL expression and decreased the cell proliferation in CML cells having wild-type BCR-ABL or T315I mutations in vitro and in vivo. ${ }^{108}$

Hypomethylation is also another important strategy for overcoming imatinib-resistance. Decitabine, an agent used for hypomethylation, was reported to be effective in imatinibresistant CML cells. Phase I and phase II studies revealed that treatment of imatinib-resistant CML patients with decitabine resulted in a high hematologic response. ${ }^{109,110}$

\section{Conclusion}

In summary, imatinib is the first-line treatment for CML. However, it does not appear to be capable of eliminating all leukemia cells in patients. Acquired as well as preexisting resistance to the drug have been observed. To minimize and overcome these problems, novel strategies, such as dose escalation, drug combination therapy, and use of novel BCRABL inhibitors, have been developed.

\section{Acknowledgment}

We thank Professor Dr Anne Frary for critically reviewing the manuscript.

\section{Disclosure}

We, the authors of the manuscript, do not have any conflicts of interest.

\section{References}

1. Inokuchi K. Chronic myelogenous leukemia: from molecular biology to clinical aspects and novel targeted therapies. J Nihon Med Sch. 2006;73(4):178-192.

2. Chandra HS, Heistekamp NC, Hungerford A, et al. Philadelphia Chromosome Symposium: commemoration of the 50th anniversary of the discovery of the Ph chromosome. Cancer Genet. 2011;204(4): 171-179.

3. Chen Y, Peng C, Li D, Li S. Molecular and cellular bases of chronic myeloid leukemia. Protein Cell. 2010;1(2):124-132.

4. Groffen J, Stephenson JR, Heisterkamp N, de Klein A, Bartram CR, Grosveld G. Philadelphia chromosomal breakpoints are clustered within a limited region, bcr, on chromosome 22. Cell. 1984;36(1): 93-99.

5. Shtivelman E, Lifshitz B, Gale RP, Canaani E. Fused transcript of $a b l$ and $b c r$ genes in chronic myelogenous leukaemia. Nature. 1985; 315(6020):550-554.

6. Quintás-Cardama A, Cortes J. Molecular biology of bcr-abll-positive chronic myeloid leukemia. Blood. 2009;113(8):1619-1630.

7. Deininger MV, Goldman JM, Melo JV. The molecular biology of chronic myeloid leukemia. Blood. 2000;96(10):3343-3356.

8. Kantarjian HM, Giles F, Quintás-Cardama A, Cortes J. Important therapeutic targets in chronic myelogenous leukemia. Clin Cancer Res. 2007;13(4):1089-1097.

9. Ren R. Mechanisms of BCR-ABL in the pathogenesis of chronic myelogenous leukaemia. Nat Rev Cancer. 2005;5(3):172-183.

10. Pendergast AM, Quilliam LA, Cripe LD, et al. BCR-ABL-induced oncogenesis is mediated by direct interaction with the SH2 domain of the GRB-2 adaptor protein. Cell. 1993;75(1):175-185.

11. Ilaria RL Jr, Van Etten RA. P210 and P190(BCR/ABL) induce the tyrosine phosphorylation and DNA binding activity of multiple specific STAT family members. $J$ Biol Chem. 1996;271(49):31704-31710.

12. Chai SK, Nichols GL, Rothman P. Constitutive activation of JAKs and STATs in BCR-Abl-expressing cell lines and peripheral blood cells derived from leukemic patients. J Immunol. 1997;159(10): $4720-4728$.

13. Sillaber C, Gesbert F, Frank DA, Sattler M, Griffin JD. STAT5 activation contributes to growth and viability in Bcr/Abl-transformed cells Blood. 2000;95(6):2118-2125.

14. Skorski T, Kanakaraj P, Nieborowska-Skorska M, et al. Phosphatidylinositol-3 kinase activity is regulated by $\mathrm{BCR} / \mathrm{ABL}$ and is required for the growth of Philadelphia chromosome-positive cells. Blood. 1995;86(2):726-736.

15. Naughton R, Quiney C, Turner SD, Cotter TG. Bcr-Abl-mediated redox regulation of the PI3K/AKT pathway. Leukemia. 2009;23(8): $1432-1440$

16. Sattler M, Salgia R, Okuda K, et al. The proto-oncogene product p120CBL and the adaptor proteins CRKL and c-CRK link c-ABL, $\mathrm{p} 190 \mathrm{BCR} / \mathrm{ABL}$ and $\mathrm{p} 210 \mathrm{BCR} / \mathrm{ABL}$ to the phosphatidylinositol-3 kinase pathway. Oncogene. 1996;12(4):839-846.

17. Franke TF, Kaplan DR, Cantley LC. PI3K: downstream AKTion blocks apoptosis. Cell. 1997;88(4):435-437.

18. Zou X, Rudchenko S, Wong K, Calame K. Induction of c-myc transcription by the v-Abl tyrosine kinase requires Ras, Rafl, and cyclindependent kinases. Genes Dev. 1997;11(5):654-662.

19. Li F, Sethi G. Targeting transcription factor NF-kappaB to overcome chemoresistance and radioresistance in cancer therapy. Biochim Biophys Acta. 2010;1805(2):167-180.

20. Jagani Z, Singh A, Khosravi-Far R. FoxO tumor suppressors and BCRABL-induced leukemia: a matter of evasion of apoptosis. Biochim Biophys Acta. 2008;1785(1):63-84. 
21. Neviani P, Santhanam R, Trotta R, et al. The tumor suppressor PP2A is functionally inactivated in blast crisis CML through the inhibitory activity of the BCR/ABL-regulated SET protein. Cancer Cell. 2005;8(5): 355-368.

22. Schiffer CA. BCR-ABL tyrosine kinase inhibitors for chronic myelogenous leukemia. N Engl J Med. 2007;357(3):258-265.

23. Torgerson SR, Haddad RY, Atallah E. Chronic myelogenous leukemia for primary care physicians. Dis Mon. 2012;58(4):168-176.

24. Vardiman J, Harris NL, Brunning RD. The World Health Organization (WHO) classification of the myeloid neoplasms. Blood. 2002;100(7): 2292-2302.

25. Hehlmann R, Heimpel H, Hasford J, et al. Randomized comparison of interferon-alpha with busulfan and hydroxyurea in chronic myelogenous leukemia. The German CML Study Group. Blood. 1994;84(12): 4064-4077.

26. Talpaz M, Kantarjian H, Kurzrock R, Trujillo JM, Gutterman JU. Interferon-alpha produces sustained cytogenetic responses in chronic myelogenous leukemia. Philadelphia chromosome-positive patients. Ann Intern Med. 1991;114(7):532-538.

27. Henkes M, van der Kuip H, Aulitzky WE. Therapeutic options for chronic myeloid leukemia: focus on imatinib (Glivec, Gleevectrade mark). Ther Clin Risk Manag. 2008;4(1):163-187.

28. Guglielmi C, Arcese W, Dazzi F, et al. Donor lymphocyte infusion for relapsed chronic myelogenous leukemia: prognostic relevance of the initial cell dose. Blood. 2002;100(2):397-405.

29. Lugo TG, Pendergast AM, Muller AJ, Witte ON. Tyrosine kinase activity and transformation potency of bcr-abl oncogene products. Science. 1990;247(4946):1079-1082.

30. Zimmermann J, Caravatti G, Mett H, et al. Phenylamino-pyrimidine (PAP) derivatives: a new class of potent and selective inhibitors of protein kinase C (PKC). Arch Pharm (Weinheim). 1996;329(7):371-376.

31. Zimmermann J, Buchdunger E, Mett H, Meyer T, Lydon NB. Potent and selective inhibitors of the Abl kinase: phenylamino-pyrimidine (PAP) derivatives. Bioorg Med Chem Lett. 1997;7:187-192.

32. Savage DG, Antman KH. Imatinib mesylate - a new oral targeted therapy. N Engl J Med. 2002;346(9):683-693.

33. Schindler T, Bornmann W, Pellicena P, Miller WT, Clarkson B, Kuriyan J. Structural mechanism for STI-571 inhibition of abelson tyrosine kinase. Science. 2000;289(5486):1938-1942.

34. Druker BJ, Tamura S, Buchdunger E, et al. Effects of a selective inhibitor of the Abl tyrosine kinase on the growth of Bcr-Abl positive cells. Nat Med. 1996;2(5):561-566.

35. Buchdunger E, Zimmermann J, Mett H, et al. Selective inhibition of the platelet-derived growth factor signal transduction pathway by a protein-tyrosine kinase inhibitor of the 2-phenylaminopyrimidine class. Proc Natl Acad Sci U S A. 1995;92(7):2558-2562.

36. Buchdunger E, Zimmermann J, Mett H, et al. Inhibition of the Abl protein-tyrosine kinase in vitro and in vivo by a 2-phenylaminopyrimidine derivative. Cancer Res. 1996;56(1):100-104.

37. Beran M, Cao X, Estrov Z, et al. Selective inhibition of cell proliferation and BCR-ABL phosphorylation in acute lymphoblastic leukemia cells expressing Mr 190,000 BCR-ABL protein by a tyrosine kinase inhibitor (CGP-57148). Clin Cancer Res. 1998;4(7):1661-1672.

38. Gambacorti-Passerini C, le Coutre P, Mologni L, et al. Inhibition of the ABL kinase activity blocks the proliferation of BCR/ABL+ leukemic cells and induces apoptosis. Blood Cells Mol Dis. 1997;23(3):380-394.

39. Deininger MW, Goldman JM, Lydon N, Melo JV. The tyrosine kinase inhibitor CGP57148B selectively inhibits the growth of BCR-ABLpositive cells. Blood. 1997;90(9):3691-3698.

40. Dan S, Naito M, Tsuruo T. Selective induction of apoptosis in Philadelphia chromosome-positive chronic myelogenous leukemia cells by an inhibitor of BCR - ABL tyrosine kinase, CGP 57148. Cell Death Differ. 1998;5(8):710-715.

41. Holtz MS, Slovak ML, Zhang F, Sawyers CL, Forman SJ, Bhatia R. Imatinib mesylate (STI571) inhibits growth of primitive malignant progenitors in chronic myelogenous leukemia through reversal of abnormally increased proliferation. Blood. 2002;99(10):3792-3800.
42. Appel S, Boehmler AM, Grünebach F, et al. Imatinib mesylate affects the development and function of dendritic cells generated from CD34+ peripheral blood progenitor cells. Blood. 2004;103(2):538-544.

43. Daley GQ, Van Etten RA, Baltimore D. Induction of chronic myelogenous leukemia in mice by the P210bcr/abl gene of the Philadelphia chromosome. Science. 1990;247(4944):824-830.

44. Zhang X, Ren R. Bcr-Abl efficiently induces a myeloproliferative disease and production of excess interleukin-3 and granulocytemacrophage colony-stimulating factor in mice: a novel model for chronic myelogenous leukemia. Blood. 1998;92(10):3829-3840.

45. Wolff NC, Ilaria RL Jr. Establishment of a murine model for therapytreated chronic myelogenous leukemia using the tyrosine kinase inhibitor STI571. Blood. 2001;98(9):2808-2816.

46. Druker BJ, Talpaz M, Resta DJ, et al. Efficacy and safety of a specific inhibitor of the BCR-ABL tyrosine kinase in chronic myeloid leukemia. N Engl J Med. 2001;344(14):1031-1037.

47. Druker BJ, Sawyers CL, Kantarjian H, et al. Activity of a specific inhibitor of the BCR-ABL tyrosine kinase in the blast crisis of chronic myeloid leukemia and acute lymphoblastic leukemia with the Philadelphia chromosome. N Engl J Med. 2001;344(14):1038-1042.

48. Sawyers CL, Hocchaus A, Feldman E, et al. Imatinib induces hematologic and cytogenetic responses in patients with chronic myelogenous leukemia in myeloid blast crisis: results of a phase II study. Blood. 2002;99(10):3530-3539.

49. Kantarjian HM, Vellekoop L, McCredie KB, et al. Intensive combination chemotherapy (ROAP 10) and splenectomy in the management of chronic myelogenous leukemia. J Clin Oncol. 1985;3(2):192-200.

50. Kantarjian HM, Keating MJ, Talpaz M, et al. Chronic myelogenous leukemia in blast crisis. Analysis of 242 patients. Am J Med. 1987;83(3): 445-454.

51. Kantarjian HM, Keating MJ, Estey EH, et al. Treatment of advanced stages of Philadelphia chromosome-positive chronic myelogenous leukemia with interferon-alpha and low-dose cytarabine. J Clin Oncol. 1992;10(5):772-778.

52. Ottmann OG, Druker BJ, Sawyers CL, et al. A phase 2 study of imatinib in patients with relapsed or refractory Philadelphia chromosome-positive acute lymphoid leukemias. Blood. 2002;100(6): 1965-1971.

53. Wassmann B, Klein SA, Scheuring U, et al. Hematologic and cytogenetic remission by STI571(Glivec) in a patient relapsing with accelerated phase CML after second allogeneic stem cell transplantation. Bone Marrow Transplant. 2001;28(7):721-724.

54. Talpaz M, Silver RT, Druker BJ, et al. Imatinib induces durable hematologic and cytogenetic responses in patients with accelerated phase chronic myeloid leukemia: results of a phase 2 study. Blood. 2002;99(6): 1928-1937.

55. Guilhot $\mathrm{F}$, Chastang $\mathrm{C}$, Michallet $\mathrm{M}$, et al. Interferon alfa-2b combined with cytarabine versus interferon alone in chronic myelogenous leukemia. French Chronic Myeloid Leukemia Study Group. $N$ Engl J Med. 1997;337(4):223-229.

56. O'Brien SG, Guilhot F, Larson RA, et al. Imatinib compared with interferon and low-dose cytarabine for newly diagnosed chronic-phase chronic myeloid leukemia. N Engl J Med. 2003;348(11):994-1004.

57. Shah NP, Nicoll JM, Nagar B, et al. Multiple BCR-ABL kinase domain mutations confer polyclonal resistance to the tyrosine kinase inhibitor imatinib (STI571) in chronic phase and blast crisis chronic myeloid leukemia. Cancer Cell. 2002;2(2):117-125.

58. Deininger M, O’Brien SG, Guilhot F, et al. International randomized study of interferon vs STI571 (IRIS) 8-year follow-up: sustained survival and low risk for progression or events in patients with newly diagnosed chronic myeloid leukemia in chronic phase treated with imatinib [ASH Annual Meeting Abstract]. Blood. 2009;114: Abstr 1126

59. Kantarjian H, Talpaz M, O'brien S, et al. Survival benefit with imatinib mesylate therapy in patients with accelerated-phase chronic myelogenous leukemia - comparison with historic experience. Cancer. 2005; 103(10):2099-2108. 
60. Kantarjian HM, Talpaz M, O’Brien S, et al. Survival benefit with imatinib mesylate versus interferon-alpha-based regimens in newly diagnosed chronic-phase chronic myelogenous leukemia. Blood. 2006; 108(6):1835-1840.

61. Kantarjian HM, O’Brien S, Cortes J, et al. Imatinib mesylate therapy improves survival in patients with newly diagnosed Philadelphia chromosome-positive chronic myelogenous leukemia in the chronic phase: comparison with historic data. Cancer. 2003;98(12):2636-2642.

62. Kantarjian H, O'Brien S, Garcia-Manero G, et al. Very long-term follow-up results of imatinib mesylate therapy in chronic phase chronic myeloid leukemia after failure of interferon alpha therapy. Cancer. 2012;118(12):3116-3122.

63. Kantarjian H, O'Brien S, Cortes J, et al. Survival advantage with imatinib mesylate therapy in chronic-phase chronic myelogenous leukemia (CML-CP) after IFN-alpha failure and in late CML-CP, comparison with historical controls. Clin Cancer Res. 2004;10(1 Pt 1):68-75.

64. Tanaka MF, Kantarjian H, Cortes J, Ohanian M, Jabbour E. Treatment options for chronic myeloid leukemia. Expert Opin Pharmacother. 2012;13(6):815-828.

65. National Comprehensive Cancer Network (NCCN) Clinical Practice Guidelines in Oncology: Chronic Myeloid Leukemia. 2010. Fort Washington: NCCN; 2010. Available from: http://www.24hmb.com/UpLoad/ Editor/2010/1/4/2010010471144545.pdf. Accessed October 20, 2012.

66. Baccarani M, Cortes J, Pane F, et al; European LeukemiaNet. Chronic myeloid leukemia: an update of concepts and management recommendations of European LeukemiaNet. J Clin Oncol. 2009;27(35): 6041-6051.

67. Hughes TP, Kaeda J, Branford S, et al; International Randomised Study of Interferon versus STI571 (IRIS) Study Group. Frequency of major molecular responses to imatinib or interferon alfa plus cytarabine in newly diagnosed chronic myeloid leukemia. $N$ Engl J Med. 2003;349(15):1423-1432.

68. Marin D, Milojkovic D, Olavarria E, et al. European LeukemiaNet criteria for failure or suboptimal response reliably identify patients with CML in early chronic phase treated with imatinib whose eventual outcome is poor. Blood. 2008;112(12):4437-4444.

69. Alvarado Y, Kantarjian H, O’Brien S, et al. Significance of suboptimal response to imatinib, as defined by the Europoean LeukemiaNet, in the long-term outcome of patients with early chronic myeloid leukemia in chronic phase. Cancer. 2009;115(16):3709-3718.

70. Kantarjian HM, O'Brien S, Cortes JE, et al. Complete cytogenetic and molecular responses to interferon-alpha-based therapy for chronic myelogenous leukemia are associated with excellent long-term prognosis. Cancer. 2003;97(4):1033-1041.

71. Druker BJ, Guilhot F, O’Brien SG, et al; IRIS Investigators. Five-year follow-up of patients receiving imatinib for chronic myeloid leukemia. N Engl J Med. 2006;355(23):2408-2417.

72. Hughes TP, Hochhaus A, Branford S, et al; IRIS Investigators. Long-term prognostic significance of early molecular molecular response to imatinib in newly diagnosed chronic myeloid leukemia: an analysis from the International Randomized Study of Interferon and STI571 (IRIS). Blood. 2010;116(19):3758-3765.

73. Kantarjian HM, Shan J, Jones D, et al. Significance of increasing levels of minimal residual disease in patients with Philadelphia chromosomepositive chronic myelogenous leukemia in complete cytogenetic response. J Clin Oncol. 2009;27(22):3659-3663.

74. Baccarani M, Saglio G, Goldman J, et al; European LeukemiaNet. Evolving concepts in the management of chronic myeloid leukemia: recommendations from an expert panel on behalf of the European LeukemiaNet. Blood. 2006;108(6):1809-1820.

75. Marin D, Ibrahim AR, Lucas C, et al. Assessment of BCR-ABL1 transcript levels at 3 months is the only requirement for predicting outcome for patients with chronic myeloid leukemia treated with tyrosine kinase inhibitors. J Clin Oncol. 2012;30(3):232-238

76. Quintas-Cardama A, Kantarjian HM, Cortes JE, et al. Mechanisms of primary and secondary resistance to imatinib in chronic myeloid leukemia. Cancer Control. 2009;16(2):122-131.
77. Melo JV, Chuah C. Resistance to imatinib mesylate in chronic myeloid leukaemia. Cancer Lett. 2007;249(2):121-132.

78. Branford S. Chronic myeloid leukemia: molecular monitoring in clinical practice. Hematology Am Soc Hematol Educ Program. 2007: 376-383.

79. Borthakur G, Kantarjian HM, O'Brien SM, et al. Efficacy of dasatinib in patients with previously untreated chronic myeloid leukemia in early chronic phase (CML-CP). J Clin Oncol. 2008;26: Abstr 7013.

80. Jabbour E, Hochhaus A, Coutre P, et al. Minimal cross-intolerance between nilotinib and imatinib in patients with imatinibintolerant chronic myelogenous leukemia (CML) in chronic phase (CP) or accelerated phase (AP). J Clin Oncol. 2008;26: Abstr 7063.

81. Cortes J, O'Brien S, Jones D, et al. Efficacy of nilotinib (formerly AMN107) in patients with newly diagnosed, previously untreated Philadelphia chromosome-positive chronic myelogenous leukemia in early chronic phase (CML-CP). Blood. 2008;112:Abstr 446.

82. Agrawal M, Garg RJ, Cortes J, Quintás-Cardama Al. Tyrosine kinase inhibitors: the first decade. Curr Hematol Malig Rep. 2010;5(2): 70-80.

83. Kantarjian HM, Hochhaus A, Saglio G, et al. Nilotinib versus imatinib for the treatment of patients with newly diagnosed chronic phase, Philadelphia chromosome-positive, chronic myeloid leukaemia: 24-month minimum follow-up of the phase 3 randomised ENESTnd trial. Lancet Oncol. 2011;12(9):841-851.

84. Kantarjian HM, Shah NP, Cortes JE, et al. Dasatinib or imatinib in newly diagnosed chronic-phase chronic myeloid leukemia: 2-year follow-up from a randomized phase 3 trial (DASISION). Blood. 2012;119(5): 1123-1129.

85. Deremer DL, Ustun C, Natarajan K. Nilotinib: a second-generation tyrosine kinase inhibitor for the treatment of chronic myelogenous leukemia. Clin Ther. 2008;30(11):1956-1975.

86. Golemovic M, Verstovsek S, Giles F, et al. AMN107, a novel aminopyrimidine inhibitor of Bcr-Abl, has in vitro activity against imatinib-resistant chronic myeloid leukemia. Clin Cancer Res. 2005; 11(13):4941-4947.

87. Weisberg E, Manley PW, BreitensteinW, et al. Characterization of AMN107, a selective inhibitor of native and mutant Bcr-Abl. Cancer Cell. 2005;7(2):129-141.

88. Walz C, Sattler M. Novel targeted therapies to overcome imatinib mesylate resistance in chronic myeloid leukemia (CML). Crit Rev Oncol Hematol. 2006;57(2):145-164.

89. Sattler M, Mohi MG, Pride YB, et al. Critical role for Gab2 in transformation by BCR/ABL. Cancer Cell. 2002;1(5):479-492.

90. Martinelli G, Martelli AM, Grafone T, et al. A new Abl kinase inhibitor (AMN107) has in vitro activity on $\mathrm{CML} \mathrm{Ph}+$ blast cells resistant to imatinib [ASH Annual Meeting Abstract]. Blood. 2004;104:Abstr 4687.

91. Kantarjian H, Giles F, Wunderle L, et al. Nilotinib in imatinib-resistant CML and Philadelphia chromosome-positive ALL. $N$ Engl J Med. 2006;354(24):2542-2551.

92. Lockton JA, Smethurst D, Macpherson M, et al. Phase I ascending single and multiple dose studies to assess the safety, tolerability and pharmacokinetics of AZD0530, a highly selective, dual-specific Src-Abl inhibitor [ASCO Meeting Abstract]. J Clin Oncol. 2005;23(16S):3125.

93. O'HareT, Pollock R, Stoffregen EP, et al. Inhibition of wild-type and mutant Bcr-Abl by AP23464, a potent ATP-based oncogenic protein kinase inhibitor: implications for CML. Blood. 2004;104(8): 2532-2539.

94. Gambacorti-Passerini C, Gasser M, Ahmed S, Assouline S, Scapozza L. Abl inhibitor BMS354825 binding mode in Abelson kinase revealed by molecular docking studies. Leukemia. 2005;19(7):1267-1269.

95. Lombardo LJ, Lee FY, Chen P, et al. Discovery of N-(2-chloro6-methyl- phenyl)-2-(6-(4-(2-hydroxyethyl)- piperazin-1-yl)-2methylpyrimidin-4-ylamino)thiazole-5-carboxamide (BMS-354825), a dual Src/Abl kinase inhibitor with potent antitumor activity in preclinical assays. J Med Chem. 2004;47(27):6658-6661. 
96. Tokarski J, Newitt J, Lee FY, et al. The crystal structure of Abl kinase with BMS-354825, a dual SRC/ABL kinase inhibitor [ASH Annual Meeting Abstract]. Blood. 2004;104:Abstr 553.

97. Shah NP, Tran C, Lee FY, Chen P, Norris D, Sawyers CL. Overriding imatinib resistance with a novel ABL kinase inhibitor. Science. 2004;305(5682):399-401.

98. Talpaz M, Shah NP, Kantarjian H, et al. Dasatinib in imatinibresistant Philadelphia chromosome-positive leukemias. N Engl J Med. 2006;354(24):2531-1541.

99. Branford S, Hughes T, Nicoll J, et al. Molecular responses and mutation analysis in imatinib resistant patients with $\mathrm{Ph}$ positive leukemia treated with the dual src/abl kinase inhibitor BMS-354825. 10th Congress of the European Hematology Association. Stockholm; 2005.

100. Gumireddy K, Baker SJ, Cosenza SC, et al. A non-ATP-competitive inhibitor of BCR-ABL overrides imatinib resistance. Proc Natl Acad Sci US A. 2005;102(6):1992-1997.

101. Kimura S, Naito H, Segawa H, et al. NS-187, a potent and selective dual Bcr-Abl/Lyn tyrosine kinase inhibitor, is a novel agent for imatinib-resistant leukemia. Blood. 2005;106(12):3948-3954.

102. Harrington EA, Bebbington D, Moore J, et al. VX-680, a potent and selective small-molecule inhibitor of the Aurora kinases, suppresses tumor growth in vivo. Nat Med. 2004;10(3):262-267.

103. Young MA, Shah NP, Chao LH, et al. Structure of the kinase domain of an imatinib-resistant Abl mutant in complex with the Aurora kinase inhibitor VX-680. Cancer Res. 2006;66(2):1007-1014.
104. Cortes J, Albitar M, Thomas D, et al. Efficacy of the farnesyl transferase inhibitor R1 15777 in chronic myeloid leukemia and other hematologic malignancies. Blood. 2003;101(5):1692-1697.

105. Cortes J, Garcia-Manero G, O’Brien S, et al. Phase I study of a imatinib and tipifarnib (Zarnestra2, R115777) in patients with chronic myeloid leukemia in chronic phase refractory to imatinib [ASH Annual Meeting Abstract]. Blood. 2003;102:Abstr 3383.

106. Borthakur G, Kantarjian H, Daley G, et al. Pilot study of lonafarnib, a farnesyl transferase inhibitor, in patients with chronic myeloid leukemia in the chronic or accelerated phase that is resistant or refractory to imatinib therapy. Cancer. 2006;106(2):346-352.

107. Mayerhofer M, Aichberger KJ, Florian S, et al. Identification of mTOR as a novel bifunctional target in chronic myeloid leukemia: dissection of growth-inhibitory and VEGF-suppressive effects of rapamycin in leukemic cells. FASEB J. 2005;19(8):960-962.

108. Lu Z, Jin Y, Qiu L, Lai Y, Pan J. Celastrol, a novel HSP90 inhibitor, depletes Bcr-Abl and induces apoptosis in imatinib-resistant chronic myelogenous leukemia cells harboring T315I mutation. Cancer Lett. 2010;290(2):182-191.

109. Kantarjian HM, O’Brien S, Cortes J, et al. Results of decitabine (5-aza2'deoxycytidine) therapy in 130 patients with chronic myelogenous leukemia. Cancer. 2003;98(3):522-528.

110. Issa JP, GharibyanV, Cortes J, et al. Phase II study of low-dose decitabine in patients with chronic myelogenous leukemia resistant to imatinib mesylate. J Clin Oncol. 2005;23(17):3948-3956.

\section{Publish your work in this journal}

The Journal of Blood Medicine is an international, peer-reviewed, open access, online journal publishing laboratory, experimental and clinical aspects of all topics pertaining to blood based medicine including but not limited to: Transfusion Medicine; Blood collection, Donor issues, Transmittable diseases, and Blood banking logistics; Immunohematology; Artificial and alternative

\section{Dovepress}

blood based therapeutics; Hematology; Biotechnology/nanotechnology of blood related medicine; Legal aspects of blood medicine; Historical perspectives. The manuscript management system is completely online and includes a very quick and fair peer-review system. Visit http://www.dovepress.com/ testimonials.php to read real quotes from published authors. 